\title{
Perfectly conducting loop of wire moving through a uniform and stationary magnetic field
}

(O movimento de uma espira condutora perfeita através de um campo magnético estacionário e uniforme)

\author{
Fernando L. da Silveira ${ }^{\mathbb{W}}$, Ives S. Araujo ${ }^{1}$, Maria C. Varriale ${ }^{2}$, Luiz F. Ziebell ${ }^{1}$ \\ ${ }^{1}$ Instituto de Física, Universidade Federal do Rio Grande do Sul, Porto Alegre, RS, Brazil \\ ${ }^{2}$ Instituto de Matemática, Universidade Federal do Rio Grande do Sul, Porto Alegre, RS, Brazil
}

Recebido em 4/4/2012; Aceito em 23/6/2012; Publicado em 18/2/2013

\begin{abstract}
A conductive loop of wire moving with constant velocity in a magnetic field is one of the most used examples in physics textbooks in connection to the Faraday-Lenz law, highlighting the consistency of electromagnetism with the principle of conservation of energy. It is frequently concluded that currents are not induced in a perfectly conducting loop because an induced current would have to be infinite and therefore would violate the principle of energy conservation. In this paper we discuss this problem and show that a model that includes the loop self-inductance prevents the divergence of the current and leads to results compatible with the conservation of energy.

Keywords: Faraday-Lenz law, auto-inductance, electrical resistance, perfect conductor.

Uma espira condutora movendo-se com velocidade constante através de uma região com um campo magnético

constitui-se em uma das situações mais utilizadas em livros didáticos de física em conexão com a lei de FaradayLenz, servindo para evidenciar a consistência do eletromagnetismo com o princípio da conservação da energia. Da análise dessa situação é frequentemente extraída a conclusão de que correntes não são induzidas em espiras perfeitamente condutoras porque nestas uma corrente induzida teria de ser infinita e portanto violaria o princípio de conservação de energia. No presente artigo apresentamos uma discussão sobre este problema e mostramos que um modelo para a espira que inclua sua autoindutância não apresenta divergência da corrente e leva a resultados compatíveis com a conservação da energia.
\end{abstract}

Palavras-chave: lei de Faraday-Lenz, autoindução, resistência elétrica, condutor.

\section{Introduction}

The electromagnetic (EM) theory, set forth in the XIX century as a coherent body of knowledge, is one of the greatest intellectual achievements of the human history. It unified electrical and magnetic phenomena, settled the ground for the understanding of the light as an electromagnetic wave, and lead to the conception that EM waves do not need a material support in order to propagate. Significant part of our present technology is directly related to practical applications of the EM theory, and the understanding about the inner works of any electronic product requires basic knowledge of fundamental concepts of the EM theory. The theory deals with vector fields $\mathbf{E}$ and $\mathbf{B}$, which satisfy a set of mathematical laws known as the Maxwell laws, which are

\footnotetext{
${ }^{1}$ E-mail: lang@if.ufrgs.br.

extensively studied in undergraduate courses in physics and engineering. However, despite the usual emphasis on the study of these very important laws of natural science, some features remain insufficiently explored in textbook treatments.

In the present paper we discuss some of these insufficiently explored features, related to a simple example which is usual in textbooks on electromagnetism as illustration of the relationship between the time variation of the magnetic flux through the area limited by a closed circuit and the induced eletromotive force (emf). The example considers a rectangular conducting loop of wire, with sides $h$ and $w$ and electrical resistance $R$, moving with constant velocity $\mathbf{v}$ through a rectangular region $a b c d$, which is perpendicular to a uniform and stationary magnetic field $\mathbf{B}$ (Fig. 1). 


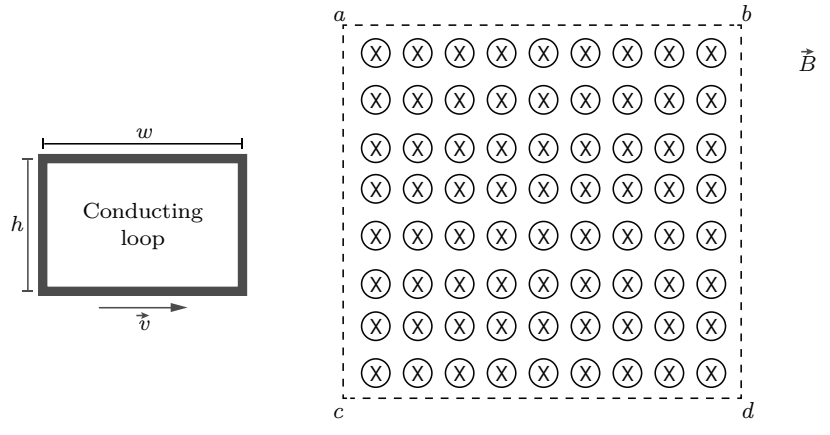

Figure 1 - Conducting loop of wire before entering a region with uniform and stationary magnetic field.

It can be easily shown that an induced emf $\varepsilon$ occurs in the loop, starting when it initiates the penetration in the $a b c d$ region and vanishing when it is completely inside this region. The emf starts again when the loop starts to leave the abcd region and vanishes when it leaves the region. The absolute value of the emf is

$$
|\varepsilon|=B h v,
$$

and the intensity of the induced electric current is

$$
|i|=\frac{B h v}{R} .
$$

Figure 2 shows the induced current as a function of the position of the leading side of the moving loop, considering $x=0$ the position in which the loop starts the penetration of the $a b c d$ region, and $x_{1}=\overline{a b}$ the position in which the loop starts to leave this region. The magnitude of the current is the same in the entrance and in the exit phases, but the sense of the current is inverted, as described by the Lenz law.

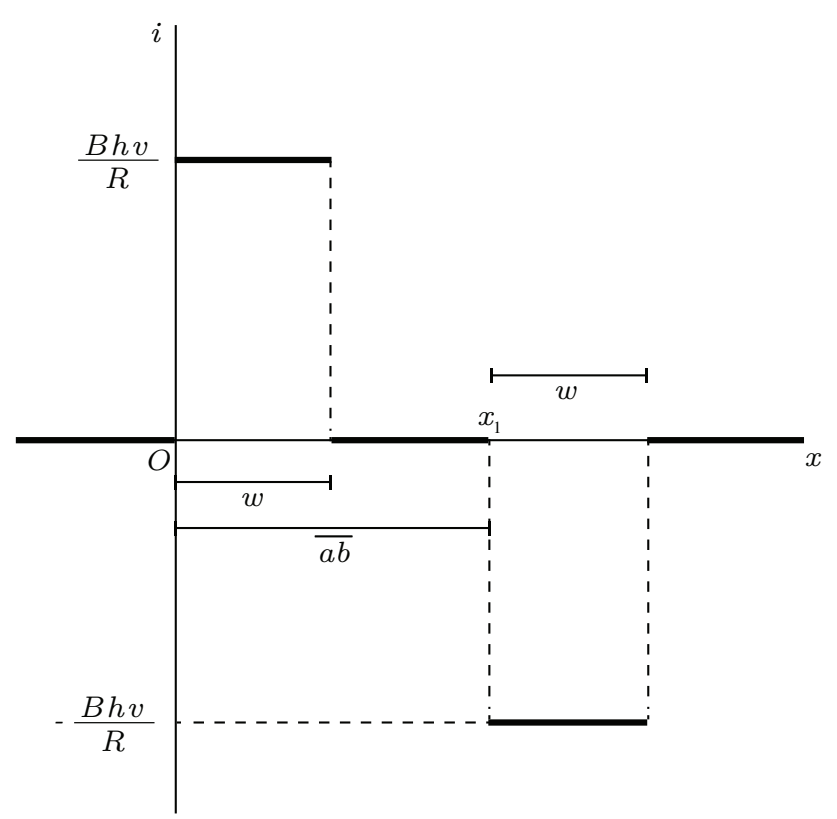

Figure 2 - Induced current as a function of the position of the leading side of the moving loop, in the case of a purely resistive loop.
As a consequence of the induced current the loop suffers a magnetic force whose magnitude is given by

$$
\left|\mathbf{F}_{B}\right|=\frac{B^{2} h^{2} v}{R}
$$

both in the entrance phase and in the exit phase. The magnetic force is opposite to the sense of motion of the loop, in both phases. For the movement of the loop to be maintained at constant speed, some agent external to the system loop+magnetic field must apply a force of the same magnitude as the magnetic force, but in opposite sense. That is, the external force must point in the same sense as the velocity of the loop. The external agent therefore introduces power into the system, given by

$$
P_{F}=\frac{B^{2} h^{2} v^{2}}{R}
$$

On the other hand, power is dissipated in the loop, by the so-called Joule effect, in an amount given by

$$
P_{R}=R i^{2}=\frac{B^{2} h^{2} v^{2}}{R} .
$$

These results show that the power introduced into the system by the external agent, given by Eq. (四), is exactly the same as the power dissipated by Joule effect, given by Eq. (国). The conclusion, always emphasized by the textbooks, is that the Faraday-Lenz law is consistent with the principle of energy conservation.

This textbook example clearly shows that the induced currents, the forces and the powers involved, increase when the electrical resistance of the loop is reduced. An interesting variation of the situation can therefore be proposed: What happens if the electrical resistance of the loop is reduced until vanishing, that is, if the loop is made from a perfectly conducting material?

Well known textbooks discuss this limiting situation using arguments which may be understood erroneously, if interpreted as simple extrapolation of the result given by Eq. (四). For instance, in the book by Tipler and Mosca (2004, p. 923) [प]:

... there can be no electric field in a superconducting ring because it has no resistance, so a finite electric field would drive an infinite current. The flux through the ring is thus frozen and cannot change.

Similar statement can be found in the book by Chabay and Sherwood (2010, p. 963-964) [च]:

Suppose that you try to change the flux through a superconductor ring. This changing flux would be associated with a curly electric field (...). This curly electric field would drive an infinite current in the ring, because there is no resistance! 
Statements like these may easily appear to have been obtained by direct use of Eq. (四). However, Eqs. (অ) to (国) are not strictly valid when the electrical resistance becomes very small, leading to divergence in the limit of vanishing resistance. The divergence does not occur in the physical phenomena, it is rather consequence of the use of a model which does not take into account the inductance of the loop. In the following sections we discuss some particular cases and demonstrate that the divergences in Eqs. (四)-(四) do not occur if a more complete model is utilized for the description of the loop. For the analysis, we assume that the emission of electromagnetic radiation is negligible in all cases considered.

The problem of the conducting loop, either resistive or with vanishing resistance, taking into account the self-inductance, has been discussed in a paper by Saslow [3], which develops and discuss the pertinent differential equations. Another and more recent analysis appears in Ref. [四], which discusses the occurrence of self-inductive effects in the context of perfectly conducting and superconducting circuits, rather than resistive circuits, and intends to be useful to motivate the interest of students on the topic of superconductivity. The present paper adopts a perspective somewhat complementary to that of Ref. [四]. It does not intend to discuss the superconducting state, but offers a detailed discussion on the transition between circuits which can be considered as purely resistive and circuits with vanishing resistance, with the discussion restrained to the use of concepts which are usual in general physics classes. The approach which is proposed in the present paper avoids the use of second-order differential equations, relying instead on a mathematical approach more consistent with that of textbooks on general physics, like those already mentioned [四, ఐ] .

The structure of the paper is the following: in section 2 we discuss the case of a conducting loop of wire moving with constant velocity, taking into account the self-inductance and the resistance of the loop. In sub-section 2.1 we present the limiting case of a perfectly conducting loop, still taking into account the selfinductance. The transition between the perfectly conducting case, in which the resistance vanishes and the self-inductance can not be neglected, and the opposite case in which the self-inductance is negligible and the loop can be considered exclusively resistive, is discussed in more detail in sub-section 2.2. Section 3 presents the case of a loop of wire moving exclusively under the effect of a magnetic field, without forces produced by external agents. Some numerical results and estimations appear in section 4. Finally, conclusions and final remarks appear in section 5 .

\section{Resistive and inductive loop moving with constant velocity}

The textbook example discussed in the introduction section is based on a model which can be called the resistive model, in which the self-inductance of the loop is neglected. The use of the resistive model implies the instantaneous appearing of an induced current with constant intensity, as described by Eq. (『). The instantaneous response is clearly the result of an approximation, since in the actual case the current must vary smoothly when there are changes in the emf.

The point is that in the derivation of Eq. (四) the current transient due to self-inductive effects has been neglected. If the self-inductance $L$ is taken into account, and considering the constant emf $\varepsilon=B h v$, the induced current is a time-dependent quantity,

$$
i(t)=\frac{B h v}{R}\left(1-e^{-t /(L / R)}\right),
$$

the well-known solution of the initial value problem for the $R L$ circuit,

$$
L \frac{d i}{d t}+R i=B h v, \quad i(0)=0 .
$$

If the time constant $\tau=L / R$ is very small compared to the time intervals which are relevant, for instance the interval for the complete entrance of the loop in the abcd region, the duration of the transient current becomes negligible, and the constant value of current predicted by the resistive model becomes an useful approximation.

Equation ([) is well defined for finite values of $R$ and $L$, because it is a solution of a differential equation describing a circuit with constant emf and non-vanishing resistive and inductive characteristics, Eq. (ㅁ). The limiting case in which the inductance $L$ is made to vanish amounts to that briefly considered in section 1 . The case in which the resistance $R$ can be considered vanishing is considered in the following sub-section.

\subsection{The case of a perfectly conducting and in- ductive loop}

Here we consider the case of a loop of wire with resistance $R=0$ and finite self-inductance $L$. The loop moves with constant velocity and is incident on a region with uniform magnetic field, as depicted in Fig. $\mathbf{\square}$. A constant emf is produced while the loop enters the region abcd. The initial value problem describing the situation is obtained from Eq. (प), simply as follows

$$
L \frac{d i}{d t}=B h v, \quad i(0)=0 .
$$

whose solution is given by

$$
i(t)=\frac{B h v}{L} t .
$$


The induced current can be written in terms of the position of the leading side of the moving loop, $x=v t$, considering $x=0$ the position of entrance in the abcd region,

$$
i(x)=\frac{B h}{L} x
$$

It is seen that the induced current grows linearly with the distance of penetration into the region where there is a uniform magnetic field, attaining maximum value for $x=w$, i.e., when the loop is entirely in the abcd region. It is clear that there is no divergence in the induced electric current in the case of a perfectly conducting loop of wire, differently from what would be obtained with use of Eq. (『).

The magnetic force resisting the entering loop is therefore proportional to the distance of penetration,

$$
F_{B}=\frac{B^{2} h^{2} x}{L}
$$

as well as the motive power developed by the external agent,

$$
P_{F}=\frac{B^{2} h^{2} v}{L} x
$$

To discuss the conservation of energy, we evaluate the rate of variation of the magnetic energy associated to the loop with inductance $L$,

$$
P_{B}=\frac{d}{d t}\left(\frac{L i^{2}}{2}\right)=L i \frac{d i}{d t}=\frac{B^{2} h^{2} v}{L} x,
$$

where in the last step we have used Eq. (四) for $i(t)$.

It is seen that the rate of variation of the magnetic energy during the phase in which the loop is entering the abcd region, given by Eq. ([त्]), is exactly the same as the power provided by the external agent, Eq. (एव), indicating that at least in the entrance phase the purely inductive case also satisfies the principle of energy conservation.

After the moment in which the loop is totally inserted into the region $a b c d$, the magnetic flux over the area of the loop is constant and therefore the induced emf vanishes. However, because the electrical resistance is zero, the electric current remains unchanged. On the other hand, when the loop starts to leave the region, the magnetic flux starts to decrease, and it is easy to show that the current linearly decreases, vanishing when the loop leaves completely the abcd region. The current along the whole transit of a perfectly conducting loop through the abcd region is shown in Fig. B, as a function of the position of the leading side of the loop.

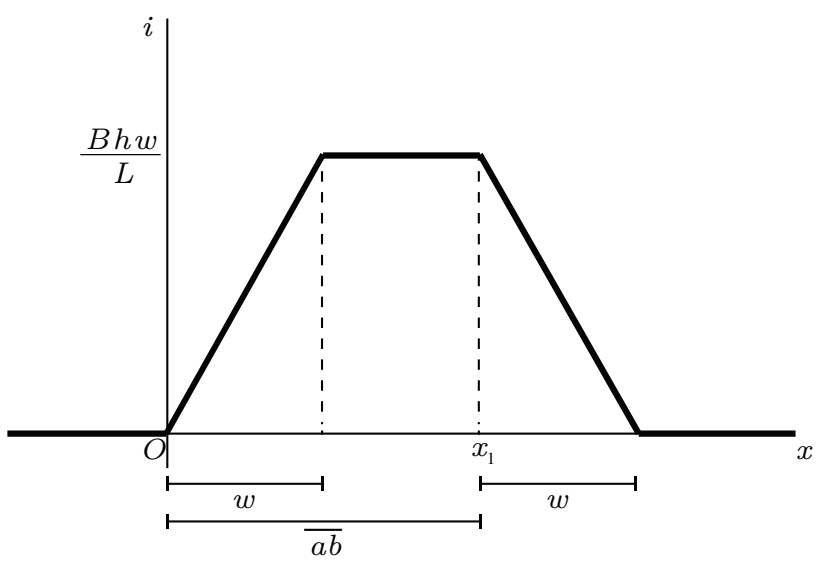

Figure 3 - Induced current as a function of the position of the leading side of the moving loop, in the case of a purely inductive loop.

Figures $\square$ and $\mathbf{\square}$ are noticeably different. Part of the difference which is visually noticeable is related to the inversion of the sense of current propagation which happens in the case of the purely resistive loop, depicted in Fig. 2. Due to the inversion in the sense of the current, the resistive force is opposite to the loop velocity, both in the entrance and in the exit of the region abcd. The external agent makes positive work in both phases of the motion, and the total work done is equal to the energy dissipated by Joule effect, as seen in Eqs. (四) and (19). In the purely inductive case, on the other hand, the magnetic force is opposite to the velocity when the loop is entering the region and in the same sense as the velocity when the loop is leaving the region. The external agent makes positive work when the loop is entering the region, in the same amount as the increase in the magnetic energy, as demonstrated by Eqs. (며) and ([ए3), and makes negative work when the loop is leaving the region, in the same amount as the decrease in the magnetic energy. The total work made by the external agent is null, and the energy conservation principle is not violated.

\subsection{Transition between the resistive and the perfectly conducting cases}

Figures $\square$ and $\mathbf{\square}$ show the behavior of the electrical current when the loop is exclusively resistive and when it is exclusively inductive, respectively. These two cases may be understood as limiting situations for the general case of a loop with resistive and inductive properties. This more general case is illustrated in Fig. \#, which shows the current as a function of time, as predicted by Eq. ([), for four inductive and resistive loops which enter the $a b c d$ region with the same velocity. To fit the behavior of the current over a long time range, the scales in the two axis are not linear. For the four loops represented, the self-inductance is the same, while their electrical resistances have the same ratios as 1:2:5:10. The initial growth of the current, independently of the 
time constant $\tau=L / R$, is exactly the same that happens in the case of a perfectly conducting loop, whose behavior is described by Eq. (四), and is represented by the dashed straight line passing through the origin. It is seen that, when the electric resistance tends to zero, the current simply tends to grow continuously, without divergence, while the flux due to the external magnetic field is increasing over the loop area.

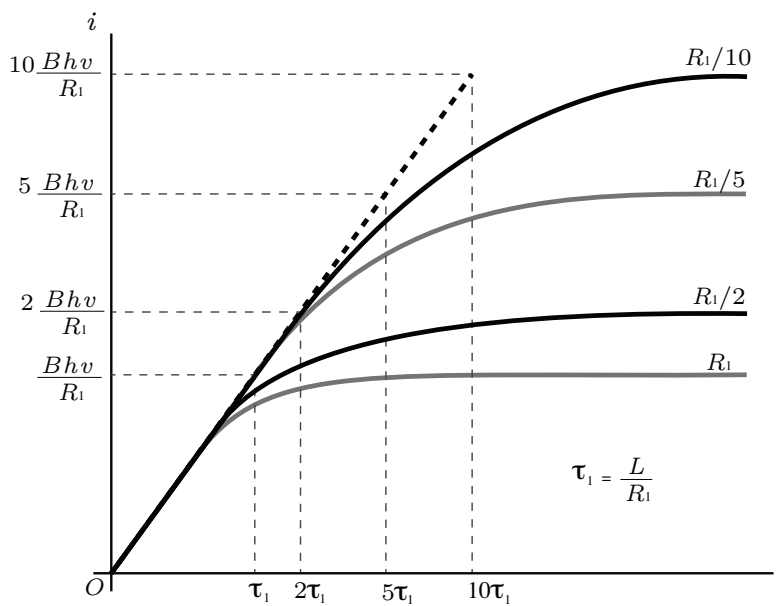

Figure 4 - Intensity of the induced current as a function of time after the entrance in the region of uniform magnetic field, for four loops with the same self-inductance and different values of the resistance.

In fact, it is easy to verify that the limit of Eq. (四) for $R \rightarrow 0$ is Eq. (四). Using Eq. (四) and expanding the exponential function in powers of the non-dimensional quantity $(R t / L)$, we obtain

$$
i(t)=B h v\left(\frac{t}{L}-R \frac{t^{2}}{L^{2}}+\ldots\right) \rightarrow \frac{B h v}{L} t,
$$

where the last result is obtained in the limit $R \rightarrow 0$.

Moreover, for finite $R$, the first order approximation shows that the current is proportional to $t$ for small values of $t$. That is exactly what is seen in Fig. 四. For any value of $R$, the initial rate of current variation is the same as the rate of variation for the case of perfectly conducting loop, given by Eq. (四).

On the other hand, for finite $R$ and for $t \rightarrow \infty$, Eq. (四) predicts that the limiting value of the current is inversely proportional to $R$, as in Eq. (『), since

$$
\lim _{t \rightarrow \infty} e^{-R t / L}=0
$$

The curves depicted in Fig. $⿴$ confirm that the limiting value of the current increases for decreasing electrical resistance. The time for attaining the limiting value of the current also increases for decreasing resistance, because the time constant is proportional to $R^{-1}$.

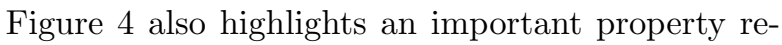
lated to the time constant $\tau$, namely, that at $t=\tau$ the current attains nearly $63 \%$ of its maximum value, which would be attained at such a time for linear growth.

\section{Perfectly conducting loop moving exclusively under the effect of a mag- netic field}

In this section we discuss a situation which has a fundamental difference relative to the cases discussed in the previous section. Here we consider a perfectly conducting moving loop which does not suffer the action of external forces. Only magnetic forces are in action when the loop enters or leaves the abcd region. From an energetic point of view the kinetic energy of the loop decreases when the loop enters the region, due to the magnetic force opposed to the sense of motion, and occurs a corresponding increase in magnetic energy. Since there is no dissipation in a perfect conductor, and since electromagnetic radiation is assumed to be negligible, the summation of kinetic energy $E_{c}$ and magnetic energy $E_{B}$ is a constant,

$$
E_{c}+E_{B}=\frac{1}{2} m v^{2}+\frac{1}{2} L i^{2}=\frac{1}{2} m v_{0}^{2},
$$

where $v_{0}$ is the initial speed of the loop.

From Eq. (四), assuming $v=v(t)$,

$$
L \frac{d i}{d t}=B h v(t), \quad i(0)=0 .
$$

Remembering that $v(t)=d x / d t$, Eq. (ㅁ]) can be written as follows

$$
d i=\frac{B h}{L} d x, \quad i(x=0)=0,
$$

whose solution is given by

$$
i=\frac{B h}{L} x .
$$

This result is exactly the same as obtained in Eq. (ㅁ) . It is therefore demonstrated that, even for variable loop velocity due to the effect of the magnetic force, the intensity of the induced current grows linearly with the distance of penetration of the leading front of the loop into the abcd region.

If the loop velocity vanishes before the loop is completely inside the abcd region, the magnetic force sends the loop moving back, reflecting it from the region with the magnetic field, so that the loop recovers the initial kinetic energy, but moving in opposite sense. For that to occur, the length of the segment along the motion must be larger than the distance of maximal penetration in the region $a b c d$, namely $w>X$, where $X$ is the point where the kinetic energy vanishes,

$$
\frac{1}{2} m v_{0}^{2}=\frac{1}{2} L i^{2}(X) .
$$

Using Eq. (एप), condition (एव]) be put in the following form,

$$
\frac{1}{2} m v_{0}^{2}=\frac{1}{2} L\left(\frac{B h X}{L}\right)^{2}
$$


which leads to the following expression for the distance of maximum penetration into the abcd region,

$$
X=\frac{\sqrt{m L}}{B h} v_{0} .
$$

Consequently, the condition for occurrence of reflection from the abcd region is as follows,

$$
w>\frac{\sqrt{m L}}{B h} v_{0} .
$$

If condition (ㅍ.: is not satisfied, the loop enters completely in the abcd region, despite the breaking magnetic force. Therefore, it emerges in the opposite side. When leaving the region, the magnetic force is in the same sense as the motion, and the current decreases and finally vanishes, when the loop has recovered the initial velocity.

The conclusion is that, due to the absence of dissipation in the perfectly conducting loop, the loop recovers the initial kinetic energy, no matter if it suffers reflection from the abcd region or if it crosses through that region. Similar result was found in the analysis of the motion of a magnet passing through a superconducting tube [回].

\section{Some numerical results}

For further comparison between the cases of purely resistive and purely inductive loops moving with constant velocity under the influence of an external agent, we produce some numerical estimates, considering two square loops with side $h$, made from a cylindrical wire with transverse radius $r$, with $r<h$. We assume $h=5.0 \mathrm{~cm}$ and $r=1.0 \mathrm{~mm}$. The magnitude of the magnetic field in the abcd region is assumed to be $0.05 \mathrm{~T}$. For order of magnitude estimate we assume that the mass density of the wire is near that of copper, $\rho_{m} \simeq 8.9 \times 10^{3} \mathrm{~kg} / \mathrm{m}^{3}$, and that the resistivity of the wire in the resistive case is nearly that of copper, $\rho \simeq 1.7 \times 10^{-8} \Omega \cdot \mathrm{m}$. by

The electric resistance of the resistive loop is given

$$
R=\rho \frac{4 h}{\pi r^{2}},
$$

and the inductance of the square loops is given by [G]

$$
L \simeq \frac{2 \mu_{0} h}{\pi}\left[\ln \left(\frac{h}{r}\right)-0.774\right],
$$

where $\mu_{0}$ is the magnetic permeability of the vacuum. For the values of parameters which we have assumed, $R \simeq 1.1 \times 10^{-3} \Omega$ and $L \simeq 1.3 \times 10^{-7} \mathrm{H}$.

Assuming that the inductance can be neglected, i.e., assuming the validity of the resistive model, we use Eq. (『) and obtain the magnitude of the current in the purely resistive loop,

$$
\left|i_{R}\right|=\frac{B v \pi r^{2}}{4 \rho} .
$$

On the other hand, the maximum magnitude of the current in the purely inductive loop can be obtained from Eq. (四),

$$
\left|i_{L}\right|_{\text {max. }}=\frac{B \pi h}{2 \mu_{0}[\ln (h / r)-0.774]},
$$

where Eq. (2. has been used for the inductance of the loop.

The ratio between the current intensities in the two limiting cases, obtained from Eq. (ए7) divided by

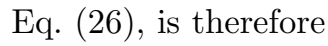

$$
\frac{\left|i_{L}\right|_{\max .}}{\left|i_{R}\right|}=\frac{2 \rho h}{\mu_{0} v r^{2}[\ln (h / r)-0.774]} \simeq \frac{416}{v} .
$$

Considering the loop speed $v \simeq 1.0 \mathrm{~m} / \mathrm{s}$, this rough estimate shows that the maximum value of the current in the perfectly conducting loop is nearly 400 times the intensity of the current in the purely resistive loop. The current intensities in the perfectly conducting and in the resistive loop, Eqs. (ㅍ) and ([2]), would be respectively $\simeq 1000 \mathrm{~A}$ and $\simeq 2.3 \mathrm{~A}$. This simple example shows that the perfectly conducting loop with inductive properties, despite avoiding the divergences predicted when the inductance is neglected, may indeed feature currents which are much more intense than the current in a resistive loop with the same geometry.

We also make numerical estimates of the conditions for which it is reasonable to neglect the effects of selfinductance for a moving loop of wire, considering typical parameters. As discussed in the paragraph following Eq. (प), the inductive effects can be neglected in the case of a resistive loop if the time constant $\tau=L / R$ is very small compared with a typical time interval. For the parameters which we have assumed for the resistive loop, $\tau \simeq 1.2 \times 10^{-4} \mathrm{~s}$. As a relevant time scale it is possible to use the time for the loop to enter or to leave the $a b c d$ region, given by $h / v$. The condition for relevance of self-inductance effects, therefore, can be cast as follows

$$
v \geq \frac{5.0 \times 10^{-2}}{1.2 \times 10^{-4}} \simeq 416 \frac{\mathrm{m}}{\mathrm{s}} .
$$

In the case of loop speed $v=1 \mathrm{~m} / \mathrm{s}$, as assumed in the previous paragraph, self-inductive effects can be neglected for resistive loops. Even for $v=10$ or $20 \mathrm{~m} / \mathrm{s}$, relatively high values for laboratory demonstration, self-inductive effects can be safely neglected.

Finally, we discuss on the conditions which determine whether a perfectly conducting loop of wire moving without the influence of an external agent is reflected or enters completely into the region $a b c d$. Solving Eq. ([2: for $v_{0}$, remembering that we are considering $w=h$, we obtain that the loop is reflected if the initial velocity satisfies the condition

$$
v_{0}<\frac{B h^{2}}{\sqrt{m L}} .
$$

For the parameters which we have assumed, the perfectly conducting loop is reflected if $v_{0}<4.6 \mathrm{~m} / \mathrm{s}$. 


\section{Final remarks}

In the present work we have used basic concepts of electromagnetic theory to discuss what happens with a perfectly conducting loop of wire moving through a region with a uniform and stationary magnetic field. The study was motivated by the observation that even wellknown textbooks may convey the notion that induced currents do no appear in such loops, since the appearing of a current would violate the principle of energy conservation [ $[\mathbf{D}$ ]. Using a mathematical approach consistent with that of textbooks on general physics, we have tried to show that such reasoning is not correct, being a consequence of neglecting the self-inductance of the loop, which can not be neglected when the electric resistance is negligible.

In the discussion of the problem we have considered the case of a loop with resistive and inductive properties moving with constant velocity, with emphasis on the limiting cases of purely resistive and purely inductive loop, and also the case of a loop moving without influence of a external agent. In the latter case, the velocity of the loop varies due to the interaction with the magnetic field. The theoretical analysis has been illustrated by some numerical estimates.

Our analysis has used a particular physical situation to emphasize the general feature that conclusions which are spurious and incompatible with observations can be obtained from theoretical analysis, if limited modeling is utilized for description of situations which are beyond the range of validity of a model, and that more accurate modeling can put the analysis back in consonance with observations. Other similar situations can be found in different physical contexts. For instance, some analogy can be found with a mechanical situation, related to the inertia of a body. The current does not diverge in a perfectly conducting loop of wire under the effect of an induced eletromotive force, due to the self-inductance, in the same way as the velocity of a body does not change instantly under the effect of a force, due to the body inertia.

The bottom-line is that the phenomena which have been discussed offer excellent opportunity for discussion of basic features of electromagnetic theory, and also opportunity for discussions on the limitations of models which are utilized for description of physical systems. A model will always be a simplified representation of reality, so that the results obtained with use of the model are valid only within a limited context $[\mathbb{\square}-\mathbf{g}]$.

\section{Acknowledgments}

L.F.Z. acknowledge support from Brazilian agency CNPq. The authors are grateful to Dr. P. Pureur Neto for motivating discussions and useful suggestions.

\section{References}

[1] P.A. Tipler and G. Mosca, Physics for Scientists and Engineers: Extended Version (E.H. Freeman and Co., New York, 2004), 5th ed..

[2] R.W. Chabay and B.A. Sherwood, Matter and Interactions: Volume 2: Electric and Magnetic Interactions (John Wiley, 2010), 3rd. ed.

[3] W.M. Saslow, American Journal of Physics 55, 986 (1987).

[4] O.F. Schilling and S.S. Sugui Jr., European Journal of Physics 25, 337 (2004).

[5] Y. Levin and F.B. Rizzato, Physical Review E 74, 066605 (2006).

[6] http://emclab.mst.edu/inductance/square.html

[7] R.S. Justi and J.K. Gilbert, International Journal of Science Education 24, 369 (2002).

[8] M. Develaki, Science and Education 16, 725 (2007).

[9] R.V. Brandão, I.S. Araujo, and E.A. Veit, A Física na Escola 9, 10 (2008). 